Brief paper

\title{
Reset passivation of nonlinear controllers via a suitable time-regular reset map ${ }^{\text {* }}$
}

\author{
Fulvio Forni ${ }^{\mathrm{a}, *}$, Dragan Nešić ${ }^{\mathrm{b}}$, Luca Zaccarian ${ }^{\mathrm{a}}$ \\ a DISP, University of Roma, Tor Vergata, Via del Politecnico 1, 00133 Roma, Italy \\ ${ }^{\mathrm{b}}$ EEE Department, University of Melbourne, Australia
}

\section{A R T I C L E I N F O}

\section{Article history:}

Received 4 May 2010

Received in revised form

11 January 2011

Accepted 15 March 2011

Available online 20 July 2011

\section{Keywords:}

Passivity

Reset control system

Hybrid system

\begin{abstract}
A B S T R A C T
For a class of square continuous-time nonlinear controllers we design a suitable resetting rule inspired by the resetting rule for Clegg integrators and First Order Reset Elements (FORE). With this rule, we prove that the arising hybrid system with temporal regularization is passive in the conventional continuous-time sense with a small shortage of input passivity decreasing with the temporal regularization constant. Based on the passivity property, we then investigate the finite gain stability of the interconnection between this passive controller and a passive nonlinear plant.
\end{abstract}

(C) 2011 Elsevier Ltd. All rights reserved.

\section{Introduction}

In recent years, much attention has been given to the analysis and design problem of control systems in the hybrid context, namely when the closed-loop dynamics obeys either a continuous law imposing a constraint on the pointwise derivative of the solution when it belongs to the so-called flow set, and/or a discrete law imposing a constraint on the jump that the solution undertakes when it belongs to the so-called jump set. This type of interpretation of hybrid systems, thereby merging classical discrete- and continuous-time concepts in a unifying framework has been pursued in the past years by providing a specific mathematical characterization of the underlying mathematical theory. An extensive survey of the corresponding results can be found in Goebel, Sanfelice, and Teel (2009). A specific instance of hybrid systems corresponds to the case analyzed here: continuoustime plants controlled by a hybrid controller, namely a hybrid closed loop where the jumps only affect the controller states. Within this class of systems a relevant example consists in the reset control systems first introduced in Clegg (1958), where a jump linear system (the "Clegg integrator") generalizing a linear integrator was proposed. This generalization was then further

\footnotetext{
The material in this paper was partially presented at the 8th IFAC Symposium on Nonlinear Control Systems (NOLCOS), September 1-3, 2010, Bologna, Italy. This paper was recommended for publication in revised form by Associate Editor Michael Malisoff under the direction of Editor Andrew R. Teel.

* Corresponding author. Tel.: +39 6 72597337; fax: +39 672597460 .

E-mail addresses: forni@disp.uniroma2.it, fulvioforni@gmail.com (F. Forni), d.nesic@ee.unimelb.edu.au (D. Nešić), zack@disp.uniroma2.it (L. Zaccarian).
}

developed in Horowitz and Rosenbaum (1975) where it was extended to first order linear filters, and therein called First Order Reset Elements (FORE). FORE received much attention in recent years and have been proven to overcome some intrinsic limitations of linear controllers (Beker, Hollot, \& Chait, 2001). Moreover, by relying on Lyapunov approaches, suitable analysis and synthesis tools for the stability of a class of reset systems generalizing control systems with FORE have been proposed in Aangenent, Witvoet, Heemels, van de Molengraft, and Steinbuch (2010), Beker, Hollot, Chait, and Han (2004), Nešić, Zaccarian, and Teel (2008) and the references therein. Moreover, in the recent paper (Carrasco, Banos, \& Van der Schaft, 2010) the $\mathscr{L}_{2}$ stability of reset control systems has been addressed in the passivity context, by showing interesting properties of the reset system under the assumption that the continuous-time part of the reset controller is passive before resets and that a suitable non-increase condition is satisfied by the storage function at jumps. In Carrasco et al. (2010), it was also shown by a simulation example that resets may help closedloop performance in passivity-based closed loops.

In this paper we further develop the ideas of Carrasco et al. (2010) by using a specific temporally regularized reset strategy for the reset controller. The reset strategy generalizes the new interpretation of FOREs and Clegg integrators proposed in Nešić et al. (2008), Zaccarian, Nešić, and Teel (2005) and the references therein. We show that, with the proposed reset strategy, passivation is possible for any continuous-time underlying dynamics under some sector growth assumption on the right-hand side of the continuous-time dynamics of the controller. The obtained passivity property is characterized by an excess of output passivity and a lack of input passivity whose size can be made arbitrarily small by suitably adjusting the reset rule. As 
an example, the proposed reset strategy allows us to establish a passivity property for any FORE, including those characterized by an exponentially unstable pole, while the results in Carrasco et al. (2010) only allow us to establish passivity of FOREs with stable poles. On the other hand, we do not address here the context of partial reset compensators studied in Carrasco et al. (2010) but we insist that the whole controller state is reset to zero. The increased potential of the reset rule proposed here in the full reset case is illustrated on a nonlinear simulation example.

The paper is organized as follows. In Section 2, we describe the proposed reset rule. In Section 3, we state a passivity result and then establish finite $\mathcal{L}_{2}$ gain properties of interconnected systems. Finally, in Section 4 we discuss a simulation example.

\section{A class of nonlinear reset controllers}

Consider the following nonlinear controller mapping the input $v$ to the output $u$,

$\dot{x}_{c}=f\left(x_{c}, v, \theta\right), \quad u=h\left(x_{c}, \theta\right)$,

where $u \in \mathbb{R}^{q}, v \in \mathbb{R}^{q}$, so that the controller is square from $v$ to $u$, and $\theta$ is an auxiliary input vector in $\mathbb{R}^{m}$. The following regularity assumption is satisfied by the right-hand side (continuity might be relaxed. See Teel (1999)).

Assumption 1. The functions $f$ and $h$ are continuous in their arguments ${ }^{1}$ and satisfy the following sector conditions: namely there exist two constants $L_{f}$ and $L_{h}$ such that for all $x_{c}, v$ and $\theta$, $\left|f\left(x_{c}, \theta\right)\right| \leq L_{f}\left(\left|x_{c}\right|+|v|\right)$ and $\left|h\left(x_{c}, \theta\right)\right| \leq L_{h}\left|x_{c}\right|$.

In this paper we propose a hybrid modification of controller (1) aimed at making it passive from $v$ to $u$, regardless of the properties of the original dynamics in (1). To avoid Zeno solutions, namely solutions that exhibit infinitely many jumps in a bounded time interval, we also embed the hybrid modification with a temporal regularization clock, imposing that the controller cannot be reset to zero before $\rho$ times after the previous reset (see also Johansson, Lygeros, Sastry, and Egerstedt (1999) and Nešić et al. (2008)). The proposed hybrid controller is given by ${ }^{2}$

$\begin{cases}\dot{x}_{c}=f\left(x_{c}, v, \theta\right) & \text { if } \tau \leq \rho \text { or } \psi(u, v) \geq 0 \\ \dot{\tau}=1 & \text { if } \tau \geq \rho \text { and } \psi(u, v) \leq 0 \\ x_{c}^{+}=0 & \\ \tau^{+}=0 & \end{cases}$
$u=h\left(x_{c}, \theta\right)$

where $\psi(u, v)$ is defined as

$\psi(u, v)=\left(u+\epsilon_{1} v\right)^{T}\left(v-\epsilon_{2} u\right)$

and $\epsilon_{1}$ and $\epsilon_{2}$ are some (typically small) non-negative scalars. As usual in the hybrid system framework, we call $C=\left\{\left(x_{c}, \tau\right.\right.$, $v, \theta): \tau \leq \rho$ or $\psi\left(h\left(x_{c}, \theta\right), v\right) \geq 0$ \} the flow set, and $D=$ $\left\{\left(x_{c}, \tau, v, \bar{\theta}\right): \tau \geq \rho\right.$ and $\left.\psi\left(h\left(x_{c}, \theta\right), v\right) \leq 0\right\}$ the jump set.

The rationale behind the reset controller (1) is illustrated in Fig. 1 where the input/output space of (2) is represented for the case $u, y \in \mathbb{R}(q=1)$. In the figure, the shaded region corresponds to the set $\psi(u, v) \geq 0$ where the system always flows, regardless of the value of $\tau$. Instead, in the remaining region, where $\psi(u, v) \leq$

\footnotetext{
1 Continuity with respect to $\theta$ of the functions in (1) is assumed to guarantee (local) existence of solutions. This condition might be relaxed by relying on alternative assumptions, e.g., following the results in Teel (1999).

2 Note that the timer variable $\tau$ in (2) can grow unbounded while in some cases it might be convenient to ensure that $\tau$ belongs to a compact set. To this aim, the flow equation of $\tau$ can be replaced by $\dot{\tau}=\min \{1,1+\rho-\tau\}$, which ensures that $\tau \in[0,1+\rho]$ at all hybrid times.
}

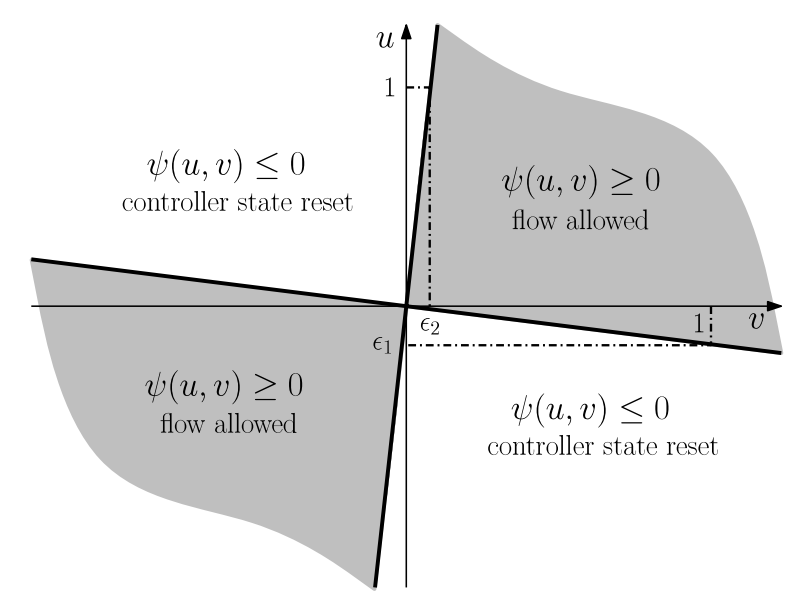

Fig. 1. Input/output space of controller (2) and subsets where $\psi(u, v) \gtreqless 0$.

0 , the system will jump provided that $\tau \geq \rho$. Note also that when $\epsilon_{1}=\epsilon_{2}=0$, the shaded region reduces to the first and the third quadrant, resembling the resetting rule characterized for the first order reset element (FORE) in Nešić et al. (2008), Zaccarian et al. (2005). When the reset occurs, since $h(0, \theta)=0$ for each $\theta \in \mathbb{R}^{m}$, the $u$ component of the input/output pair will jump to zero thus resulting in a vertical jump to the horizontal axis. Moreover, $\epsilon_{1}$ and $\epsilon_{2}$ allow us to have extra degrees of freedom in the resetting rule. In particular, the goal of $\epsilon_{1}$ is to guarantee that the reset rule maps the new input/output pair in the interior of the shaded set whenever $v \neq 0$. Instead, as it will be clear next, the goal of $\epsilon_{2}$ is to modify the resetting rule to obtain some strict output passivity for the reset controller (2). A natural interpretation of the sets in Fig. 1 is that, if we disregard temporal regularization, the phase shift between $v$ and $u$ is forced to be less than $90^{\circ}$ by way of the resetting rule. It is emphasized that augmenting the controller dynamics (1) with temporal regularization as in (2) becomes necessary when wanting to establish useful closed-loop properties of the interconnection of the controller to a continuoustime plant. Indeed, it is evident from Fig. 1 that when resetting from the origin of the $(v, u)$ plane (which always belongs to the jump set), the overall state remains unchanged (thus belonging again to the jump set). This implies that, regardless of $\epsilon_{1}$ and $\epsilon_{2}$, the system without temporal regularization admits Instantaneous Zeno solutions (namely solutions that only jump and never flow) at the origin of the $(v, u)$ plane. These solutions do not converge to zero whenever $v$ is the output of a plant (as in Section 3.2, later) where $v=0$ does not imply that the state of the plant is zero (e.g., if the plant output has smaller dimension than the plant state).

Controller (2) will be dealt with in this paper following the framework of Cai and Teel (2009), Goebel et al. (2009) and Goebel and Teel (2006). In particular, by Assumption 1, controller (2) satisfies the hybrid basic assumptions (see, e.g., Cai and Teel (2009)) which, under the hypothesis that $v$ and $\theta$ are measurable signals, ensure desirable regularity properties of the solutions, such as existence, and robustness to arbitrarily small perturbations (see Goebel et al. (2009) for details). Some standard notation related to the hybrid framework of Goebel et al. (2009), the concepts of hybrid time domains, lifted and projected signals, suitable properties of the solutions of (2), and several notions of passivity are reported in Appendix.

\section{Main results}

\subsection{Passivity of the reset controller}

The following proposition characterizes regularity of solutions to (2). Then, the subsequent theorem is our main result about almost passivity of (2). 
Proposition 1. Under Assumption 1, all the solutions of (2) are uniformly non-Zeno. Moreover, for each $\mathcal{L}_{p}$ integrable input signal $\bar{v}$, and each measurable input signal $\bar{\theta}$, each solution pair $(\xi,(v, \theta))$ is complete, where $v$ and $\theta$ are the hybrid input signals lifted from $\bar{v}$ and $\bar{\theta}$ on $\operatorname{dom} \xi$.

Proof. For a solution pair $(\xi,(v, \theta))$, define $t_{j}=\min \{t \mid(t, j) \in$ $\operatorname{dom} \xi\}$. By the definition of $C$ and $D$ in (2), given any solution pair $(\xi,(v, \theta))$ of $(2), t_{j}-t_{j-1} \geq \rho$ for all $(t, j) \in \operatorname{dom}(\xi), j \geq 2$. This implies that the uniformly non-Zeno definition in Goebel and Teel (2006) (see also Collins (2004)) is satisfied with $T=\rho$ and $J=2$. By $C \cup D=\mathbb{R}^{n} \times \mathbb{R}_{\geq 0} \times \mathbb{R}^{q} \times \mathbb{R}^{m}$ and by the measurability of both $v$ and $\theta$, dom $\xi$ is bounded only if $\xi$ blows up in finite time. Looking at the dynamics of the system in (2a), by Assumption 1, $\left|\dot{x}_{c}\right| \leq\left|f\left(x_{c}, v, \theta\right)\right| \leq L_{f}\left|x_{c}\right|+L_{f}|v|$ and $|\dot{\tau}|=1$. Therefore, if $|v|$ is $\mathcal{L}_{p}$ integrable, $|\xi|$ is bounded in any given compact subset of $\mathbb{R}_{\geq 0} \times \mathbb{N}$.

Theorem 1. Consider the hybrid controller (2) satisfying Assumption 1 and define $\varepsilon_{1}=\frac{\epsilon_{1}}{1-\epsilon_{1} \epsilon_{2}}, \varepsilon_{2}=\frac{\epsilon_{2}}{1-\epsilon_{1} \epsilon_{2}}, k_{\rho}=\rho L_{h} L_{f}$ $\max \left\{1, \rho e^{L_{f} \rho}\right\}$, and $\bar{k}_{\rho}=k_{\rho}\left(1+\varepsilon_{2} k_{\rho}\right)$. Given a $\mathcal{L}_{2}$ integrable input signal $\bar{v} \in \mathbb{R}_{\geq 0} \rightarrow \mathcal{V}$, a measurable signal $\bar{\theta} \in \mathbb{R}_{\geq 0} \rightarrow \mathbb{R}^{m}$ and a solution pair $(\xi,(v, \theta))$ to $(2)$, with $\xi(0,0)=\left[x_{0} \tau_{0}\right]^{T}$, and with $v$ and $\theta$ lifted from $\bar{v}$ and $\bar{\theta}$ on $\operatorname{dom} \xi$, then

$$
\int_{0}^{\infty} \bar{u}(t)^{T} \bar{v}(t) \mathrm{d} t \geq-\left(\varepsilon_{1}+2 \bar{k}_{\rho}\right)\|\bar{v}\|_{2}^{2}+\varepsilon_{2}\|\bar{u}\|_{2}^{2}-\frac{2 \bar{k}_{\rho}}{\rho L_{f}^{2}}\left|x_{0}\right|_{2}^{2}
$$

where the output signal $\bar{u} \in \mathbb{R}_{>0} \rightarrow \mathbb{R}^{q}$ is projected from the hybrid output signal $u$ : $\operatorname{dom} u \rightarrow \mathbb{R}^{q}$ corresponding to the solution pair $(\xi,(v, \theta))$.

Remark 1. Theorem 1 establishes an almost passivity property for (2), with a shortage of input passivity proportional to $\varepsilon_{1}$ and $\rho$. This almost passivity property is established using the norm $\|\cdot\|_{c, 2}$ (see Appendix), namely only taking into account the continuoustime nature of the hybrid solutions. This type of passivity concept is relevant because of Proposition 1 and, moreover, allows us to rely on standard passivity results (van der Schaft, 1999) to conclude properties of the closed loop between (2) and a plant, as detailed in Section 3.2.

Proof of Theorem 1. Consider an input signal $\bar{v}: \mathbb{R}_{>0} \rightarrow \mathbb{R}^{q}$ such that $\|\bar{v}\|_{2}$ is defined, a measurable input signal $\bar{\theta}: \mathbb{R}_{\geq 0} \rightarrow \mathbb{R}^{m}$, and a solution pair $(\xi,(v, \theta))=\left(\left(\xi_{x}, \xi_{t}\right),(v, \theta)\right)$ to the hybrid system (2), where $v$ and $\theta$ are the hybrid signals lifted from $\bar{v}$ and $\bar{\theta}$ on $\operatorname{dom} \xi$. By Proposition $1, \operatorname{dom} \xi$ is unbounded. Define the set $\mathcal{T}=\bigcup_{j}\left[t_{j}, t_{j}+\rho\right] \times\{j\}$ where for all $j, t_{j}$ is such that, for each $\tau \in \mathbb{R}_{>0},\left(t_{j}-\tau, j\right) \notin \operatorname{dom} \xi$. Note that, by time regularization, $\mathcal{T} \subseteq \operatorname{dom} \xi$ but $\mathcal{T}$ is not necessarily a hybrid time domain. It follows that $\forall(t, j) \in \operatorname{dom} \xi$ such that $(t, j) \notin \mathcal{T}$ we have $\xi_{\tau}(t, j)>\rho$ therefore, from the definition of the flow set, $\xi_{\tau}(t, j)>\rho$ implies $\psi(u(t, j), v(t, j)) \geq 0$, that is, $u(t, j)^{T} v(t, j)+$ $\varepsilon_{1}|v(t, j)|^{2}-\varepsilon_{2}|u(t, j)|^{2} \geq 0$, where $\varepsilon_{1}=\frac{\epsilon_{1}}{1-\epsilon_{1} \epsilon_{2}}$ and $\varepsilon_{2}=\frac{\epsilon_{2}}{1-\epsilon_{1} \epsilon_{2}}$. Therefore

$$
\begin{aligned}
& \int_{0}^{\infty} \bar{u}(t)^{T} \bar{v}(t) \mathrm{d} t=\sum_{j} \int_{t_{j}}^{t_{j+1}} u(t, j)^{T} v(t, j) \mathrm{d} t \\
& \quad=\sum_{j}\left(\int_{t_{j}}^{t_{j}+\rho} u(t, j)^{T} v(t, j) \mathrm{d} t+\int_{t_{j}+\rho}^{t_{j+1}} u(t, j)^{T} v(t, j) \mathrm{d} t\right) \\
& \quad \geq \sum_{j}\left(\int_{t_{j}}^{t_{j}+\rho} u(t, j)^{T} v(t, j) \mathrm{d} t\right.
\end{aligned}
$$

$$
\begin{gathered}
\left.\quad-\int_{t_{j}+\rho}^{t_{j+1}} \varepsilon_{1}|v(t, j)|^{2} \mathrm{~d} t+\int_{t_{j}+\rho}^{t_{j+1}} \varepsilon_{2}|u(t, j)|^{2} \mathrm{~d} t\right) \\
\geq \sum_{j}\left(\int_{t_{j}}^{t_{j}+\rho} u(t, j)^{T} v(t, j) \mathrm{d} t-\varepsilon_{2}|u(t, j)|^{2} \mathrm{~d} t\right. \\
\left.\quad-\int_{t_{j}}^{t_{j+1}} \varepsilon_{1}|v(t, j)|^{2} \mathrm{~d} t+\int_{t_{j}}^{t_{j+1}} \varepsilon_{2}|u(t, j)|^{2} \mathrm{~d} t\right) .
\end{gathered}
$$

Consider now the continuous dynamics of $x_{c}$ in (2a). By Assumption 1, we have $\left|\dot{x}_{c}\right| \leq\left|f\left(x_{c}, v, \theta\right)\right| \leq L_{f}\left|x_{c}\right|+L_{f}|v|$. Then, for $(t, j) \in\left[t_{j}, t_{j}+\rho\right] \times\{j\} \subseteq \mathcal{T}, j>0$, we have

$$
\begin{aligned}
\left|\xi_{x}(t, j)\right| & \leq \int_{t_{j}}^{t} e^{L_{f}(t-s)} L_{f}|v(s, j)| \mathrm{d} s \\
& \leq \int_{t_{j}}^{t_{j}+\rho} e^{L_{f}\left(t_{j}+\rho-s\right)} L_{f}|v(s, j)| \mathrm{d} s \\
& \leq L_{f} M_{1} \int_{t_{j}}^{t_{j}+\rho}|v(s, j)| \mathrm{d} s,
\end{aligned}
$$

where $M_{1}=\max \left\{1, e^{L_{f} \rho}\right\}$ and where there is no dependence on the initial condition by the fact that $\xi_{x}\left(t_{j}, j\right)=0$. Moreover, for $(t, 0) \in[0, \rho] \times\{0\} \subseteq \mathcal{T}$.

$$
\begin{aligned}
\left|\xi_{x}(t, 0)\right| & \leq\left(e^{L_{f} t}\left|\xi_{x}(0,0)\right|+\int_{0}^{t} e^{L_{f}(t-s)} L_{f}|v(s, j)| \mathrm{d} s\right) \\
& \leq L_{f} M_{1}\left(\frac{\left|\xi_{x}(0,0)\right|}{L_{f}}+\int_{0}^{\rho}|v(s, j)| \mathrm{d} s\right) .
\end{aligned}
$$

Thus, using the sector bound assumption on the output function $h$, it holds that $|u(t, j)| \leq L_{h}\left|\xi_{x}(t, j)\right|$ for all $(t, j) \in\left[t_{j}, t_{j}+\rho\right] \times\{j\} \subseteq$ $\mathcal{T}$. It follows that, for $j>0$,

$$
\begin{aligned}
\int_{t_{j}}^{t_{j}+\rho}|u(t, j)|^{2} \mathrm{~d} t & \leq \int_{t_{j}}^{t_{j}+\rho} L_{h}^{2} L_{f}^{2} M_{2}\left(\int_{t_{j}}^{t_{j}+\rho}|v(s, j)| \mathrm{d} s\right)^{2} \mathrm{~d} t \\
& =\rho L_{h}^{2} L_{f}^{2} M_{2}\left(\int_{t_{j}}^{t_{j}+\rho}|v(s, j)| \mathrm{d} s\right)^{2} \\
& \leq \rho^{2} L_{h}^{2} L_{f}^{2} M_{2} \int_{t_{j}}^{t_{j}+\rho}|v(s, j)|^{2} \mathrm{~d} s,
\end{aligned}
$$

where $M_{2}=\max \left\{1, e^{2 L_{f} \rho}\right\}$ and where we used Holder's integral inequality (Vidyasagar, 1993, page 274) in the last step of (5), and for $j=0$ (by completing the squares and by Holder's integral inequality)

$$
\begin{aligned}
\int_{0}^{\rho}|u(t, 0)|^{2} \mathrm{~d} t \leq & 2 \rho^{2} L_{h}^{2} L_{f}^{2} M_{2} \\
& \times\left(\frac{\left|\xi_{x}(0,0)\right|^{2}}{\rho L_{f}^{2}}+\int_{0}^{\rho}|v(s, 0)|^{2} \mathrm{~d} s\right) .
\end{aligned}
$$

In a similar way, for $j>0$

$$
\begin{aligned}
\int_{t_{j}}^{t_{j}+\rho} u(t, j)^{T} v(t, j) \mathrm{d} t & \leq L_{h} L_{f} M_{1}\left(\int_{t_{j}}^{t_{j}+\rho}|v(t, j)| \mathrm{d} t\right)^{2} \\
& \leq \rho L_{h} L_{f} M_{1} \int_{t_{j}}^{t_{j}+\rho}|v(t, j)|^{2} \mathrm{~d} t
\end{aligned}
$$

where, as above, the last inequality is obtained by using Holder's integral inequality, and for $j=0$ (by completing the squares and 
by Holder's integral inequality)

$$
\begin{aligned}
& \int_{0}^{\rho} u(t, 0)^{T} v(t, 0) \mathrm{d} t \\
& \leq L_{h} L_{f} M_{1}\left(\frac{\left|\xi_{x}(0,0)\right|}{L_{f}}+\int_{0}^{\rho}|v(t, 0)| \mathrm{d} t\right) \int_{0}^{\rho}|v(t, 0)| \mathrm{d} t \\
& \leq 2 \rho L_{h} L_{f} M_{1}\left(\frac{\left|\xi_{x}(0,0)\right|^{2}}{\rho L_{f}^{2}}+\int_{0}^{\rho}|v(t, 0)|^{2} \mathrm{~d} t\right)
\end{aligned}
$$

Define $k_{\rho}=\rho L_{h} L_{f} M_{1}=\rho L_{h} L_{f} \max \left\{1, e^{L_{f} \rho}\right\}$. Then we have: from(5) and (7), for $j>0,\left.\left|\int_{t_{j}}^{t_{j}+\rho} u(t, j)^{T} v(t, j) \mathrm{d} t-\varepsilon_{2}\right| u(t, j)\right|^{2} \mathrm{~d} t \mid \leq$ $k_{\rho}\left(1+\varepsilon_{2} k_{\rho}\right) \int_{t_{j}}^{t_{j}+\rho}|v(t, j)|^{2} \mathrm{~d} t$, and, from (6), (8), for $j=$ $0,\left.\left|\int_{0}^{\rho} u(t, 0)^{T} v(t, 0) \mathrm{d} t-\varepsilon_{2}\right| u(t, 0)\right|^{2} \mathrm{~d} t \mid \leq 2 k_{\rho}\left(1+\varepsilon_{2} k_{\rho}\right) \int_{0}^{\rho}$ $|v(t, 0)|^{2} \mathrm{~d} t+\frac{\left|\xi_{x}(0,0)\right|^{2}}{\rho L_{f}^{2}}$.

Define now $\bar{k}_{\rho}=k_{\rho}\left(1+\varepsilon_{2} k_{\rho}\right)$ then, from (4), we can say that

$$
\begin{aligned}
\int_{0}^{\infty} & \bar{u}(t)^{T} \bar{v}(t) \mathrm{d} t \geq \sum_{j>0}-\bar{k}_{\rho} \int_{t_{j}}^{t_{j}+\rho}|v(t, j)|^{2} \mathrm{~d} t \\
& -2 \bar{k}_{\rho} \int_{0}^{\rho}|v(t, 0)|^{2} \mathrm{~d} t-2 \bar{k}_{\rho} \frac{\left|\xi_{x}(0,0)\right|^{2}}{\rho L_{f}^{2}} \\
& +\sum_{j \geq 0}\left(-\int_{t_{j}}^{t_{j+1}} \varepsilon_{1}|v(t, j)|^{2} \mathrm{~d} t+\int_{t_{j}}^{t_{j+1}} \varepsilon_{2}|u(t, j)|^{2} \mathrm{~d} t\right) \\
\geq & -\bar{k}_{\rho} \int_{0}^{\rho}|v(t, 0)|^{2} \mathrm{~d} t-2 \bar{k}_{\rho} \frac{\left|\xi_{x}(0,0)\right|^{2}}{\rho L_{f}^{2}} \\
& +\sum_{j \geq 0}\left(-\bar{k}_{\rho} \int_{t_{j}}^{t_{j}+\rho}|v(t, j)|^{2} \mathrm{~d} t+\right. \\
& \left.-\int_{t_{j}}^{t_{j+1}} \varepsilon_{1}|v(t, j)|^{2} \mathrm{~d} t+\int_{t_{j}}^{t_{j+1}} \varepsilon_{2}|u(t, j)|^{2} \mathrm{~d} t\right) \\
\geq & -\bar{k}_{\rho} \int_{0}^{\rho}|v(t, 0)|^{2} \mathrm{~d} t-2 \bar{k}_{\rho} \frac{\left|\xi_{x}(0,0)\right|^{2}}{\rho L_{f}^{2}} \\
& -\left(\varepsilon_{1}+\bar{k}_{\rho}\right) \sum_{j} \int_{t_{j}}^{t_{j+1}}|v(t, j)|^{2} \mathrm{~d} t \\
= & -\left(\varepsilon_{1}+2 \bar{k}_{\rho}\right)\|\bar{v}\|_{2}^{2}+\varepsilon_{2}\|\bar{u}\|_{2}^{2}-\frac{2 \bar{k}_{\rho}}{\rho L_{f}^{2}}\left|\xi_{x}(0,0)\right|_{2}^{2} . \quad \\
& +\sum_{t_{j}}^{t_{j+1}} \varepsilon_{2}|u(t, j)|^{2} \mathrm{~d} t \\
&
\end{aligned}
$$

Remark 2. The passivity of controller (1) induced by the reset policy in (2) is robust to small variations of the dynamics of controller (1). This is based on the fact that the passivity result in Theorem 1 is inferred from the $L_{f}$ and $L_{h}$ bounds on the functions $f$ and $h$ of the controller dynamics (1) (specified in Assumption 1). Thus, small variations of the dynamics of controller (1) can be taken into account by an appropriate selection of those bounds.

Remark 3. We emphasize the generality of the controller dynamics in (1). Despite this generality, the hybrid controller (2) satisfies the dissipativity property (3) which corresponds to an almost passivity property from $v$ to $u$. Intuitively, such a generality in the controller dynamics is related to the fact that passivity is obtained primarily via a suitable selection of the jump and flow sets $D$ and $C$, which ensure that the controller state only flows in regions where a passive behavior occurs. Roughly speaking, the passive behavior of the controller can be considered as an effect of the definition of $\psi(u, v)$, that forces a particular shape of the sets $C$ and $D$ wherein the phase shift between $u$ and $v$ is forced to be below $90^{\circ}$. Following this intuition, while $\psi(u, v)$ constrains $C$ and $D$ to induce passivity, with a shortage of passivity parameterized with a (typically small) $\varepsilon_{1}$, time regularization adds some extra constraint on $C$ and $D$ that results in a shortage of passivity parameterized with $\rho$.

\subsection{Application to feedback systems}

In this section, we use the passivity theorem (van der Schaft, 1999) to establish useful stability properties of the reset controller (2) interconnected with any passive nonlinear plant ${ }^{3}$

$\dot{x}_{p}=f_{p}\left(x_{p}, u+d\right)$

$y=h_{p}(x, u+d)$,

via the negative feedback interconnection $v=w-y$, where $w$ is an external signal. In (9), $d$ is an additive disturbance acting at the plant input. The following statement directly follows from the properties of (2) established in Theorem 1.

Proposition 2. Consider the hybrid controller (2) satisfying Assumption 1 in feedback interconnection $v=w-y$ with the plant (9). For any $\epsilon_{1} \geq 0, \epsilon_{2}>0$ and $\rho>0$, given $\varepsilon_{1}$ and $\bar{k}_{\rho}$ as in (3), if the plant is output strictly passive with an excess of output passivity $\delta_{P}>\varepsilon_{1}+2 \bar{k}_{\rho}$, then the closed-loop system (2), (9) with $v=w-y$ is finite gain $\mathscr{L}_{2}$ stable from $(w, d)$ to $(u, v)$.

In Proposition 2, we require a specific excess of output passivity from the plant because we assume that the controller requires implementation with certain prescribed selections of $\epsilon_{1}$ and $\rho$. In the case where it is possible to reduce arbitrarily these two parameters, it is possible to relax the requirements of Proposition 2 as follows.

Proposition 3. Consider the hybrid controller (2) satisfying Assumption 1 in feedback interconnection $v=w-y$ with plant (9). If plant (9) is output strictly passive, then for any $\epsilon_{2}>0$, there exist small enough positive numbers $\epsilon_{1}^{*}$ and $\rho^{*}$ such that for all $\epsilon_{1} \in\left(0, \epsilon_{1}^{*}\right]$ and all $\rho \in\left[0, \rho^{*}\right]$, the closed-loop system (2), (9) with $v=w-y$ is finite gain $\mathcal{L}_{2}$ stable from $(w, d)$ to $(u, v)$.

Proof. The proposition is a straightforward consequence of Proposition 2 noting that for a fixed $\epsilon_{2}$, the lack of output passivity established in Theorem 1 decreases monotonically to zero as $\epsilon_{1}$ and $\rho$ go to zero. Then it is always possible to reduce the two parameters to match the passivity condition in van der Schaft (1999).

Both Propositions 2 and 3 either require an explicit bound on the excess of output passivity of the plant or constrain the controller parameters $\epsilon_{1}$ and $\rho$ to be small enough. An alternative solution to this is to add an extra feedforward loop to the reset controller (2), following the derivations in Khalil (2002, page 233), to guarantee that the arising reset system is very strictly passive (Appendix). To this aim, we add the feedforward term $\epsilon_{3} v$ to the output equation of (2), thus obtaining

$$
\begin{aligned}
& \left\{\begin{array}{l}
\dot{x_{c}}=f\left(x_{c}, v, \theta\right) \\
\dot{\tau}=1
\end{array} \quad \text { if } \tau \leq \rho \text { or } \hat{\psi}(\hat{u}, v) \geq 0\right. \\
& \left\{\begin{array}{l}
x_{c}^{+}=0 \\
\tau^{+}=0
\end{array} \quad \text { if } \tau \geq \rho \text { and } \hat{\psi}(\hat{u}, v) \leq 0\right. \\
& \hat{u}=h\left(x_{c}, \theta\right)+\epsilon_{3} v
\end{aligned}
$$

\footnotetext{
3 See also Carrasco et al. (2010) for a similar application of the passivity theorem to reset controllers.
} 
where $\hat{\psi}$ is defined as

$\hat{\psi}(\hat{u}, v)=\left(\hat{u}+\left(\epsilon_{1}-\epsilon_{3}\right) v\right)^{T}\left(\left(1+\epsilon_{2} \epsilon_{3}\right) v-\epsilon_{2} \hat{u}\right)$

and $\epsilon_{3}>0$ is suitably selected as specified below. With controller (10), the following result holds.

Proposition 4. Consider the hybrid controller (10) satisfying Assumption 1 in feedback interconnection $v=w-y$ with a passive plant (9). For any $\epsilon_{1} \geq 0, \epsilon_{2}>0$ and $\rho>0$, given $\varepsilon_{1}$ and $\bar{k}_{\rho}$ as in (3), if $\epsilon_{3}>\varepsilon_{1}+2 \bar{k}_{\rho}$, then the closed-loop system (10), (9) with $v=w-y$ is finite gain $\mathcal{L}_{2}$ stable from $(w, d)$ to $(u, v)$.

Proof. Define a new output $\hat{u}=u+\epsilon_{3} v$ and denote by $\overline{\hat{u}}$ the output signal projected from $\hat{u}$ on $\mathbb{R}_{\geq 0}$. Then, from (3), we have that

$$
\begin{gathered}
\int_{0}^{\infty} \overline{\hat{u}}(t)^{T} \bar{v}(t) \mathrm{d} t \geq \epsilon_{2} \int_{0}^{\infty} \bar{u}^{T} \bar{u} \mathrm{~d} t+\left(\epsilon_{3}-\varepsilon_{1}-2 \bar{k}_{\rho}\right) \int_{0}^{\infty} \bar{v}^{T} \bar{v} \mathrm{~d} t \\
\quad \geq \frac{1}{1+2 \epsilon_{2} \epsilon_{3}}\left(\epsilon_{2} \int_{0}^{\infty} \overline{\hat{u}}^{T} \overline{\hat{u}} \mathrm{~d} t+\left(\epsilon_{3}-\varepsilon_{1}-2 \bar{k}_{\rho}\right) \int_{0}^{\infty} \bar{v}^{T} \bar{v} \mathrm{~d} t\right) .
\end{gathered}
$$

It follows that $\int_{0}^{\infty} \overline{\hat{u}}(t)^{T} \bar{v}(t) \mathrm{d} t \geq \eta_{1}\|\overline{\hat{u}}\|_{2}^{2}+\eta_{2}\|\bar{v}\|_{2}^{2}$, with $\eta_{1}=$ $\frac{\epsilon_{2}}{1+2 \epsilon_{2} \epsilon_{3}}>0$ and $\eta_{2}=\frac{\epsilon_{3}-\varepsilon_{1}-2 \bar{k}_{\rho}}{1+2 \epsilon_{2} \epsilon_{3}}>0$.

Replace now the output $u$ of controller (2) with $\hat{u}=u+\epsilon_{3} v=$ $h\left(x_{c}, \theta\right)+\epsilon_{3} v$. Then, $\hat{\psi}(\hat{u}, v)$ is obtained by substituting $u=\hat{u}-\epsilon_{3} v$ in the expression of $\psi(u, v)$ of Eq. (2b). By the passivity theorem in van der Schaft (1999), Proposition 4 follows.

Remark 4. The results in this section can be seen as a generalization of the results on full reset compensators in Carrasco et al. (2010), ${ }^{4}$ where passivity techniques are used to establish finite gain $\mathscr{L}_{2}$ stability of the closed loop between passive nonlinear plants and reset controllers. When focusing on linear reset controllers such as Clegg integrators (Clegg, 1958) and First Order Reset Elements (FORE) (Beker et al., 2004), Horowitz and Rosenbaum (1975), the novelty of Theorem 1 as compared to the results in Carrasco et al. (2010) is that those results establish passivity of FORE whose underlying linear dynamics is already passive (namely FORE with stable poles). Conversely, our results of Theorem 1 apply regardless of what the underlying dynamics of the controller is. Therefore, for example, any FORE with arbitrarily large unstable poles would still become passive using the flow and jump sets characterized in (2). ${ }^{5}$ Note however that, as compared to the approach in Carrasco et al. (2010), we are using a different selection of the flow and jump sets. Indeed, in Carrasco et al. (2010) the jump set is defined as (a slight modification of) the set of measure zero $\left\{(x, v) \in \mathbb{R}^{n} \times \mathbb{R}^{q} \mid v=0\right\}$. Instead, here we enforce a jump set which essentially corresponds to half of the input/output space of controller (1).

Remark 5. Introducing resets has advantages and disadvantages, as pointed out in Aangenent et al. (2010). Indeed, the performance improvements of resets controllers with respect to continuous controllers can be shown by transient response performance as rise time, overshoots, etc. Aangenent et al. (2010), thus suggesting a twofold goal synthesis problem for reset controllers like, for example, $\mathscr{L}_{2}$ gain and transient performance. In this sense, the structure of the controller in (2) guarantees input-output dissipativity by a suitable reset policy, leaving the continuous dynamics essentially unconstrained for achieving transient or integral-type performances. The degrees of freedom in the selection of the continuous

\footnotetext{
4 However, we do not address here the partial reset compensator case considered in Carrasco et al. (2010).

5 See, e.g., Section 4 where we illustrate the use of unstable FOREs within (2).
}
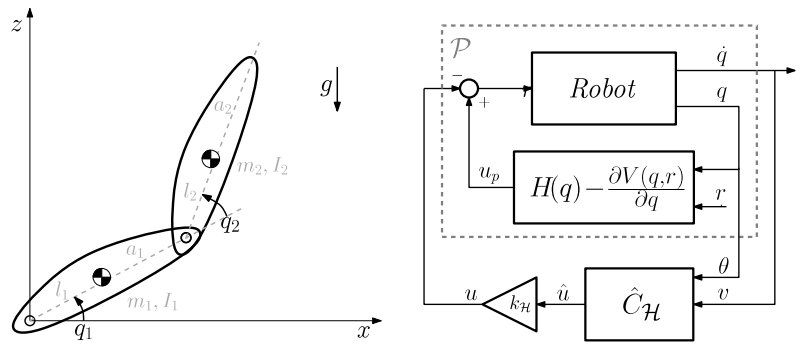

Fig. 2. The robot example.

dynamics branch out even to the possibility of using controller dynamics that exponentially destabilize the continuous-time closed loop without resets (see Section 4). It should be however noted that, as pointed out in Aangenent et al. (2010, Section 6.2), resetting in half of the input-output space of the compensator, as in (2), may negatively affect some possible "breaking action" performed by the compensator before resets. This fact might be important to keep in mind when embedding reset passivation rules. Of course, as underlined in Aangenent et al. (2010), providing fully constructive synthesis tools for reset controllers is an open research problem.

\section{Simulation example}

We consider a planar two-link rigid robot manipulator in Fig. 2, as modeled in Morabito, Nicosia, Teel, and Zaccarian (2004). Denoting by $q \in \mathbb{R}^{2}$ the two joint positions and by $\dot{q} \in \mathbb{R}^{2}$ the corresponding velocities, the manipulator is modeled as

$D(q) \ddot{q}+C(q, \dot{q}) \dot{q}+H(q)=u_{p}$

where $D(q)$ is the inertia matrix, $C(q, \dot{q}) \dot{q}$ comprises the centrifugal and Coriolis terms, $H(q)$ is the gravitational vector, and $u_{p}$ represents the external torques (see Morabito et al. (2004) for details).

Given a reference signal $r \in \mathbb{R}^{2}$ representing the desired joint position, following a standard passivity-based approach, it is possible to close a first control loop around the robot (11) to induce the equilibrium point $(q, \dot{q})=(r, 0)$ while guaranteeing passivity from a suitable input $u$ to the joint velocity output $\dot{q}$, as shown in Fig. 2. In particular, define $V(q, r)=\frac{k_{p}}{2}(q-r)^{T}(q-r)$, where the scalar $k_{p}>0$ is a weight parameter on the position error, and choose

$u_{p}=-\frac{\partial V(q, r)}{\partial q}+H(q)+u$

Then, the interconnection (11), (12) corresponds to

$D(q) \ddot{q}+C(q, \dot{q}) \dot{q}+\frac{\partial V(q, r)}{\partial q}=u$

and, following similar steps to those in Fantoni, Lozano, and Spong (2000), it can be shown to be passive from $u$ to $\dot{q}$. In particular, use the storage function $E=\frac{1}{2} \dot{q}^{T} D(q) \dot{q}+V(q, r)$ to conclude $\dot{E}=$ $\dot{q}^{T} D(q) \ddot{q}+\frac{1}{2} \dot{q}^{T} \dot{D}(q) \dot{q}+k_{p}(q-r)^{T} \dot{q}=\dot{q}^{T} u+\dot{q}^{T}\left(\frac{1}{2} \dot{D}(q)-C(q, \dot{q})\right) \dot{q}=$ $\dot{q}^{T} u$.

For the outer loop, we rely on the very strictly passive controller (10) where the dynamics in (10a) is selected as

$f\left(x_{c}, v, q\right)=\left[\begin{array}{cc}\lambda_{1} & -2 \lambda_{2} \\ -\frac{\lambda_{1}}{4} & \lambda_{2}\end{array}\right] x_{c}+k_{v} v, \quad h\left(x_{c}, q\right)=D(q) x_{c}$,

where $\lambda_{1}, \lambda_{2} \in \mathbb{R}, k_{v} \in \mathbb{R}_{>0}$, and we have used $\theta=q$. Since $D(q)$ is the inertia matrix of the robot then, from classical arguments, there exists $c_{2}>c_{1}>0$ such that $c_{1} I \leq D(q) \leq c_{2} I$, for all $q$. Therefore, $\left|D(\theta) x_{c}\right| \leq c_{2}\left|x_{c}\right|$, namely Assumption 1 holds with $L_{h}=c_{2}$. 
Remark 6. An approximate version of the closed loop in Fig. 2 could be obtained by performing a linear filtering action on $q$ and selecting $v=\frac{s}{1+\tau s} q$ with $\tau>0$ sufficiently small. This solution would not require any measurement of the joint velocities $\dot{q}$ however its effectiveness (suggested by many encouraging simulations) is not trivial to prove. Indeed, it coincides with filtering $\dot{q}$ by the strictly proper linear filter $\frac{1}{1+\tau s}$, so that the net relative degree from $u$ to the output of this strictly proper filter is 2 and passivity cannot hold. Then, for nonlinear systems, useful properties can be established as in Isidori (1995, Section 4.7), but that approach is nontrivial here for two reasons: first, we are dealing with hybrid systems, that is, systems with impulsive/discrete behaviors, and second, Proposition 4 only establishes external stability of the closed loop.

Based on the last equation in (14), the last equation in (10a), and the interconnection $v=\dot{q}$, the closed-loop robot dynamics becomes:

$\ddot{q}=-k_{\mathscr{H}} x_{c}-D(q)^{-1}\left(C(q, \dot{q}) \dot{q}+\frac{\partial V(q, r)}{\partial q}+k_{\mathcal{H}} \epsilon_{3} \dot{q}\right)$

where, as shown in Fig. 2, we choose $u=k_{\mathscr{H}} \hat{u}$, with $k_{\mathscr{H}}$ being a positive constant. From Eq. (15) it is clear why the output equation in (14) contains a multiplication by $D(q)$ : the result is that each component of $\ddot{q}$ is proportional (via $k_{\mathcal{H}}$ ) to the corresponding component of $x_{c}$. In turn, by the choice of $f$ in (14), $x_{c}$ integrates the velocity vector $v=\dot{q}$ so that $x_{c}$ resembles some sort of position information. As a result, disregarding the additional nonlinear and coupled terms in (15) (which also affect $\ddot{q}$ ), the closed-loop dynamics (15), before the reset passivation, resemble a decoupled oscillator on each joint thereby being a good candidate for a reset control system. This intuition is confirmed by the simulations, revealing that this is a successful design strategy for controller (10). Note that this qualitative design of the reset controller, without worrying about closed-loop stability, is possible because the results of Proposition 4 hold regardless of the nonlinear dynamics in (10).

By Proposition 4, the closed-loop system (11), (12) and (10a) with $u=k_{\mathscr{H}} \hat{u}$ is finite gain $\mathcal{L}_{2}$ stable. Fig. 3 compares several simulation results for this closed loop using the constant reference signal $r=\left[\begin{array}{ll}10 & 6\end{array}\right]^{T}$ and the following values of the parameters: $k_{v}=0.1, k_{p}=100, k_{\mathscr{H}}=100$ and $\rho=0.1$. First, we select $\left(\lambda_{1}, \lambda_{2}\right)=(-2,-1)$ that guarantees an exponentially stable continuous dynamics of the controller. When resets are disabled, the position and speed outputs (namely $q$ and $\dot{q}$ ), the plant input (namely $u$ ) responses, and the value of $\psi$, correspond to the dash-dotted curves in Fig. 3. When introducing resets, the response becomes the dashed curves in the figure, where it can be appreciated that the resets improve the closed-loop response. A last simulation is carried out by selecting $\left(\lambda_{1}, \lambda_{2}\right)=(2,1)$. In this case, the continuous dynamics of the controller is unstable, the controller is not passive and it is passivated by reset. To illustrate the effect of temporal regularization, we initialize both controller states at $-5 \cdot 10^{-4}$. This results in an initial dwell time of $\rho=0.1 \mathrm{~s}$ when no resets occur with $\phi<0$ (see the bottom plot of Fig. 3).

\section{Conclusions}

We proposed a reset rule for nonlinear controllers which ensures a certain type of input/output passivity. Then, relying on the passivity theorem we concluded useful properties of control systems involving this type of reset controller. A simulation example illustrates the effectiveness of the approach.

\section{Acknowledgment}

The authors would like to thank Prof. Andrew Teel for useful suggestions.
The first and third authors were supported in part by ENEAEuratom and MIUR. The second author was supported by the Australian Research Council under the Future Fellowship.

\section{Appendix. Hybrid systems notation}

As usual for hybrid systems, the state $\xi=\left(\xi_{x}, \xi_{\tau}\right)$ either continuously flows through $C$, by following the dynamic given by $f\left(\xi_{x}, v, \theta\right)$ and 1 , or jumps from $D$ to $(x, \tau)=(0,0)$. Such alternation of jumps and flow intervals can be conveniently characterized by using a generalized notion of time, called hybrid time. By following Goebel and Teel (2006), a set $E \subseteq \mathbb{R}_{\geq 0} \times \mathbb{N}$ is a hybrid time domain if it is the union of infinitely many intervals of the form $\left[t_{j}, t_{j+1}\right] \times\{j\}$ where $0=t_{0} \leq t_{1} \leq t_{2} \leq, \ldots$, or of finitely many such intervals, with the last one possibly of the form $\left[t_{j}, t_{j+1}\right] \times\{j\},\left[t_{j}, t_{j+1}\right) \times\{j\}$, or $\left[t_{j}, \infty\right) \times\{j\}$. By following Cai and Teel (2009), we call hybrid signal each function defined on a hybrid time domain. A hybrid signal $v$ : $\operatorname{dom} v \rightarrow \mathcal{V}$ is a hybrid input if $v(\cdot, j)$ is Lebesgue measurable and locally essentially bounded for each $j$. A hybrid signal $\xi$ : $\operatorname{dom} \xi \rightarrow \mathbb{R}^{n} \times \mathbb{R}_{\geq 0}$ is a hybrid arc if $\xi(\cdot, j)$ is locally absolutely continuous, for each $j$. Under Assumption 1 , a hybrid $\operatorname{arc} \xi=\left(\xi_{x}, \xi_{\tau}\right)$ and a hybrid input $(v, \theta)$ is a solution pair $(\xi,(v, \theta))$ to the hybrid system (2) if (i) () $\operatorname{dom} \xi=\operatorname{dom}(v, \theta)$, (ii) $(\xi(0,0),(v(0,0), \theta(0,0))) \in C \cup D$, and (iii) for all $j \in \mathbb{N}$ and almost all $t$ such that $(t, j) \in \operatorname{dom} \xi$,

- $(\xi(t, j),(v(t, j), \theta(t, j))) \in C$;

- $\dot{\xi}_{x}(t, j)=f\left(\xi_{x}(t, j), v(t, j), \theta(t, j)\right), \dot{\xi}_{\tau}(t, j)=1$, and for all $(t, j) \in \operatorname{dom} \xi$ such that $(t, j+1) \in \operatorname{dom} \xi$,

- $(\xi(t, j),(v(t, j), \theta(t, j))) \in D$

- $\xi_{x}(t, j+1)=0, \xi_{\tau}(t, j+1)=0$.

We say that a solution pair $(\xi,(v, \theta))$ is complete when $\operatorname{dom} \xi$ is an unbounded set. We say that a set of solution pairs $(\xi,(v, \theta))$ is uniformly non-Zeno if there exists $T \in \mathbb{R}_{>0}$ and $J \in \mathbb{N}$ such that, for any given $(t, j),\left(t^{\prime}, j^{\prime}\right) \in \operatorname{dom} \xi$, if $\left|t-t^{\prime}\right| \leq T$ then $\left|j-j^{\prime}\right| \leq J$, that is, in any time period of length $T$, no more than $J$ jumps can occur. Note that multiple instantaneous jumps are still possible, Goebel and Teel (2006).

Any continuous-time signal $\bar{v}: \mathbb{R}_{\geq 0} \rightarrow \mathbb{R}^{q}$ can be rewritten as hybrid signal with domain $E$, for any given hybrid domain $E$. In fact, suppose that $E=\bigcup_{j=0}^{\infty}\left[t_{j}, t_{j+1}\right] \times\{j\}$ is a hybrid time domain. Then, we can define a hybrid signal $v$ lifted from $\bar{v}$ on $E$ as follows: $v(t, j)=\bar{v}(t)$ for each $(t, j) \in E$. Conversely, suppose that $(\xi,(v, \theta))$ is a solution pair to the hybrid system (2). Then, the output signal $u=h\left(\xi_{x}, \theta\right)$ is a hybrid signal and $\operatorname{dom} u=\operatorname{dom} \xi$ From $u$ we can construct a continuous-time signal $\bar{u}: \mathbb{R}_{>0} \rightarrow \mathbb{R}^{q}$ projected from $u$ on $\mathbb{R}_{\geq 0}$ as follows: $\bar{u}(t)=u(t, j)$ for each $(t, j) \in$ dom $u$ such that $(t, j+1) \notin \operatorname{dom} u$, and $\bar{u}(t)=u(t, j+1)$ otherwise. We denote with $\|\bar{v}\|_{p}$ the $\mathcal{L}_{p}$ gain of a continuous-time signal $\bar{v}$. The $\mathcal{L}_{p}$ gain of a hybrid signal $v$, related to the continuous part of its domain, will be denoted by $\|v\|_{c, p}=\left(\sum_{j=0}^{J} \int_{t_{j}}^{t_{j+1}}|v(t, j)|^{p} \mathrm{~d} t\right)^{1 / p}$. Note that for any continuous-time signal $\bar{v}$ projected from a hybrid signal $\bar{v}$ on $\mathbb{R}_{\geq 0}$, we have that $\|\bar{v}\|_{q}=\|v\|_{c, p}$. Conversely, for any hybrid signal $v$ lifted from a continuous-time signal $\bar{v}$ on a given hybrid time domain $E$, we have that $\|v\|_{c, p}=\|\bar{v}\|_{p}$.

Finally, following Carrasco et al. (2010), a system $S$ with input $u: \mathbb{R}_{\geq 0} \rightarrow \mathbb{R}^{q}$ and output $y: \mathbb{R}_{\geq 0} \rightarrow \mathbb{R}^{q}$ is passive if $\int_{0}^{\infty} u(t)^{T} y(t) \mathrm{d} t \geq \beta$, with $\beta \in \mathbb{R}$. Suppose that $\int_{0}^{\infty} u(t)^{T} y(t) \mathrm{d} t \geq$ $k_{1}\|u\|_{2}+k_{2}\|y\|_{2}$, then $S$ is input strictly passive if $k_{1}>0$ and $k_{2} \geq \overline{0}$, it is output strictly passive if $k_{1} \geq 0$ and $k_{2}>0$, and it is very strictly passive if $k_{1}>0$ and $k_{2}>0$. Following Sepulchre, Jankovic, and Kokotovic (1997), we say that $S$ has an excess of passivity from the input $u$ (from the output $y$ ) if $k_{1}>0\left(k_{2}>0\right)$, and we say that $S$ has a shortage of passivity from the input $u$ (from the output $y$ ) if $k_{1}<0\left(k_{2}<0\right)$. 

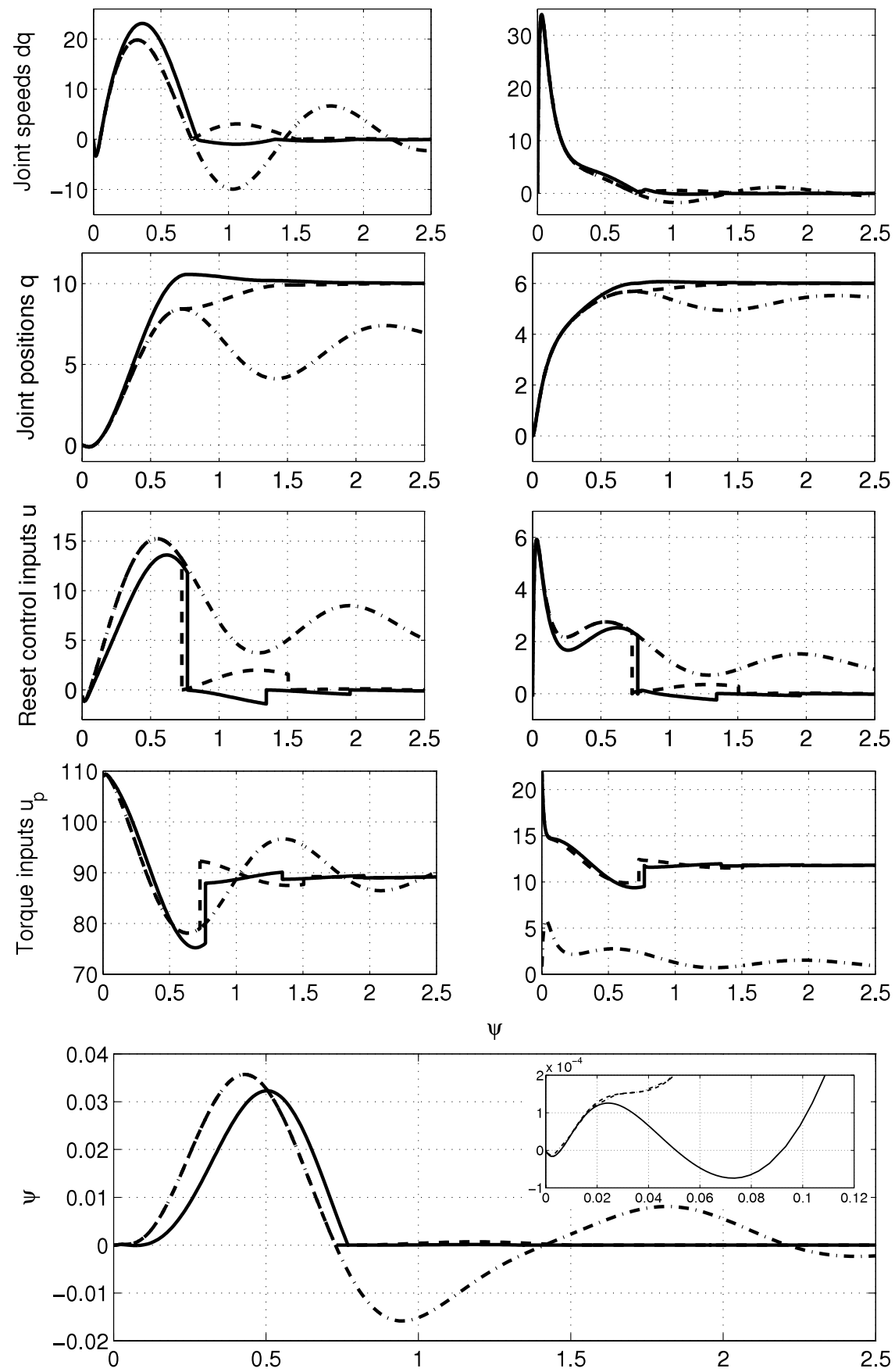

Fig. 3. Simulation results. Stable controller and no resets (dash-dotted), stable controller with resets (dashed) and unstable controller with resets (solid).

\section{References}

Aangenent, W., Witvoet, G., Heemels, W., van de Molengraft, M. J. G., \& Steinbuch, M. (2010). Performance analysis of reset control systems. International Journa of Robust and Nonlinear Control, 20(11), 1213-1233.

Beker, O., Hollot, C. V., \& Chait, Y. (2001). Plant with an integrator: an example of reset control overcoming limitations of linear feedback. IEEE Transactions Automatic Control, 46, 1797-1799.

Beker, O., Hollot, C. V., Chait, Y., \& Han, H. (2004). Fundamental properties of reset control systems. Automatica, 40, 905-915.

Cai, C., \& Teel, A. R. (2009). Characterizations of input-to-state stability for hybrid systems. Systems \& Control Letters, 58(1), 47-53.

Carrasco, J., Banos, A., \& Van der Schaft, A. (2010). A passivity-based approach to reset control systems stability. Systems $\mathcal{E}$ Control Letters, 59(1), 18-24.

Clegg, J. C. (1958). A nonlinear integrator for servomechanisms. Trans. American Institute of Electrical Engineers, 77(Part II), 41-42.

Collins, P. A trajectory-space approach to hybrid systems. In: International symposium on the mathematical theory of networks and systems, Leuven, Belgium, July 2004.
Fantoni, I., Lozano, R., \& Spong, M. W. (2000). Energy based control of the pendubot. IEEE Transactions on Automatic Control, 45(4), 725-729.

Goebel, R., Sanfelice, R., \& Teel, A. R. (2009). Hybrid dynamical systems. IEEE Control Systems Magazine, 29(2), 28-93.

Goebel, R., \& Teel, A. R. (2006). Solutions to hybrid inclusions via set and graphical convergence with stability theory applications. Automatica, 42(4), 573-587.

Horowitz, I., \& Rosenbaum, P. (1975). Non-linear design for cost of feedback reduction in systems with large parameter uncertainty. International Journal of Control, 21, 977-1001.

Isidori, A. (1995). Nonlinear control systems (3rd ed.) Springer

Johansson, K. H., Lygeros, J., Sastry, S., \& Egerstedt, M. (1999). Simulation of Zeno hybrid automata. In: Conference on decision and control (pp. 3538-3543). Phoenix, Arizona.

Khalil, H. K. (2002). Nonlinear systems (3rd ed.) USA: Prentice Hall.

Morabito, F., Nicosia, S., Teel, A. R., \& Zaccarian, L. (2004). Measuring and improving performance in anti-windup laws for robot manipulators. In B. Siciliano, A. De Luca, C. Melchiorri, \& G. Casalino (Eds.), Advances in control of articulated and mobile robots, Springer tracts in advanced robotics (pp. 61-85). chapter 3.

Nešić, D., Zaccarian, L., \& Teel, A. R. (2008). Stability properties of reset systems. Automatica, 44(8), 2019-2026. 
Sepulchre, R., Jankovic, M., \& Kokotovic, P. (1997). Constructive nonlinear control. Springer Verlag.

Teel, A. R. (1999). Asymptotic convergence from $\mathscr{L}_{p}$ stability. IEEE Transactions on Automatic Control, 44(11), 2169-2170.

van der Schaft, A. (1999). $L_{2}$-Gain and passivity in nonlinear control (2nd ed.) Secaucus, NJ, USA: Springer-Verlag New York, Inc.

Vidyasagar, M. (1993). Nonlinear systems analysis (2nd ed.) Englewood Cliffs, New Jersey: Prentice-Hall.

Zaccarian, L., Nešić, D., \& Teel, A. R. First order reset elements and the Clegg integrator revisited. In: American control conference (pp. 563-568), Portland (OR), USA, June 2005.

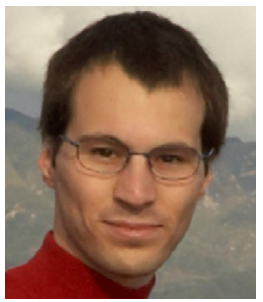

Fulvio Forni received his Ph.D degree in Computer Science and Control Engineering in 2009 from the University of Rome, Tor Vergata, where he is currently a post-doc. During 2008-2009, he spent approximatively one year as a visiting researcher at the LFCS of the University of Edinburgh, Scotland, UK and at the CCDC of the UC, Santa Barbara (USA).

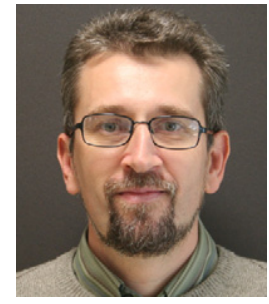

Dragan Nešić (B.S.'90, Ph.D.'97, IEEE and IEAust fellow) is a Professor in the EEE Department at The University of Melbourne, Australia. His research interests include networked control systems, discrete-time, sampled-data and continuous-time nonlinear control systems, input-tostate stability, extremum seeking control, applications of symbolic computation in control theory, hybrid control systems, and so on. He was awarded a Humboldt Research Fellowship (2003) by the Alexander von Humboldt Foundation, an Australian Professorial Fellowship (2004-2009) and Future Fellowship (2010-2014) by the Australian Research Council. He is currently a Distinguished Lecturer of CSS, IEEE (2008-2010).

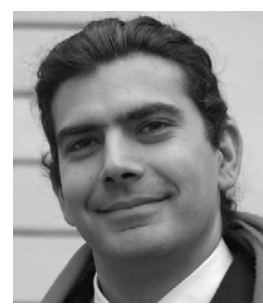

Luca Zaccarian (Laurea'95, Ph.D.'00, IEEE Senior Member) is an associate professor in control engineering at the University of Roma, Tor Vergata. His main research interests include analysis and design of nonlinear control systems, modeling and control of robots, control of thermonuclear fusion experiments and real-time control systems. He was the recipient of the 2001 O. Hugo Schuck Best Paper Award given by the American Automatic Control Council. 